actually a $G$-vector bundle since the actions of $G$ and $H$ on $G \times F$ commute. The projection $G \times F \rightarrow F$ extends by equivariances to a bundle equivalence

$$
\begin{aligned}
& G \times_{H} F \rightarrow E \\
& \downarrow \quad \downarrow \\
& G / H \approx \Omega \text {. }
\end{aligned}
$$

Hence $\pi: E \rightarrow \Omega$ is determined by the action of $H$ on $F$.

\title{
REFERENCES
}

1. R. Bott, Non-degenerate critical manifolds, Ann. of Math. (2) 60 (1954), 248-261.

2. S. Lang, Introduction to differentiable manifolds, Interscience, New York, 1962.

3. R. Palais, Morse theory on Hilbert manifolds, Topology 2 (1963), 299-340.

BRANDEIS UNIVERSITY

\section{SYMPLECTIC GROUPS OVER DISCRETE VALUATION RINGS}

BY CARL R. RIEHM ${ }^{1}$

Communicated by G. Whaples, October 12, 1964

A symplectic group over a field $\neq F_{2}$ or $F_{3}$, according to a theorem of Dickson and Dieudonné (see [1]), has no normal subgroups other than its center $\{ \pm 1\}$. Attempts at integral analogues of this theorem have of late been quite successful. First Klingenberg [6] showed that every normal subgroup of a symplectic group over a local ring is a congruence group (again with some exceptions). Then Bass, Lazard and Serre [2] showed that every normal subgroup of finite index in the symplectic group $\mathrm{Sp}_{2 n}(Z)$ over the rational integers contains a congruence subgroup if $n \geqq 2$. In [5], Jehne proved local results similar to Klingenberg's, and used them to show that any normal subgroup $G$ of the symplectic group over a suitable Dedekind ring is a congruence subgroup, if $G$ is closed under the congruence topology.

The above three integral results all assumed that the discriminant of the alternating form is a unit. The purpose of this note is to drop this restriction and give a generalization of [6]. In order to obtain a tractable canonical form, it is necessary to assume that the local

\footnotetext{
${ }^{1}$ Research partially supported by National Science Foundation grant GP-1656.
} 
ring is also a principal ideal domain, i.e., is a discrete valuation ring (and also that 2 is a unit and the residue class field $\neq F_{3}$ ). In this situation it turns out that, although every normal subgroup contains a congruence subgroup in the usual sense, a normal subgroup cannot be completely described in terms of the ordinary congruence subgroups as in [6]. A complete description is regained, however, by allowing the congruence ideal to vary from entry to entry in the matrix (see \$6). Thus a congruence subgroup in this more general sense corresponds to a square matrix, or tableau, of ideals. And when we impose certain conditions on these ideals (namely (3) in \$3), we get a 1-1 correspondence between classes of normal subgroups "of the same order" and these tableaus.

Detailed proofs for the results reported here will appear elsewhere.

1. Canonical splittings. Let $\mathrm{o}$ be a discrete valuation ring. A lattice $L$ is a free finitely generated 0 -module endowed with a nondegenerate alternating bilinear form

$$
L \times L \rightarrow \mathrm{0}
$$

which we write $(X, Y) \rightarrow X \cdot Y$ for $X, Y \in L$. Thus $X \cdot Y=-Y \cdot X, X^{2}$ $=0$ for all $X$ and $Y$ in $L$, and $X \cdot L \neq 0$ if $X \neq 0$. By a theorem of Frobenius [3, p. 79], $L$ has a canonical basis, i.e., a basis $E_{1}, \cdots, E_{2 n}$ such that $E_{i} \cdot E_{j} \neq 0$ for $i<j$ if and only if $j$ is even and $i=j-1$. Such a basis gives rise to a canonical splitting

$$
L=L_{1} \perp \cdots \perp L_{t}
$$

(orthogonal direct sum), where each $L_{i}$ has the following property: the matrix corresponding to the bilinear form on $L_{i}$ is a scalar multiple of a unimodular (alternating) matrix. This scalar generates the ideal $L_{i} \cdot L_{i}=g_{i}$. Without loss of generality, we may require that

$$
z_{1} \supset B_{2} \supset \cdots \supset z_{t}
$$

(proper containment), and it follows that $\xi_{1}, \xi_{2}, \cdots, \xi_{t}$ are unique.

2. Invariant lattices. We let $\mathrm{Sp}(L)$ denote the symplectic group of $L$, i.e., the linear automorphisms $\sigma$ of $L$ such that $\sigma X \cdot \sigma Y=X \cdot Y$ for all $X, Y \in L$. A submodule $\&$ of $L$ is called an invariant lattice if $\sigma \Omega \subseteq \Omega$ for all $\sigma \in \operatorname{Sp}(L)$.

If $\mathfrak{a}$ is an ideal, then

$$
L^{\mathfrak{a}}=\{X \in L: X \cdot L \subseteq \mathfrak{a}\}
$$

is evidently an invariant lattice. And so, if $\mathfrak{g}_{1}, \cdots, \mathfrak{g}_{t}$ are ideals,

$$
\mathfrak{R}=\mathfrak{g}_{1} L^{B_{1}}+\cdots+\mathfrak{g}_{t} L^{B_{t}}
$$


is also an invariant lattice. Conversely,

TheOREM 1. If $\mathfrak{E}$ is an invariant lattice, then there exist ideals $\mathfrak{g}_{1}, \cdots, \mathfrak{g}_{t}$ such that (2) holds and also

$$
\mathfrak{g}_{1} \subseteq \mathfrak{g}_{2} \subseteq \cdots \subseteq \mathfrak{g}_{t}, \quad \mathrm{~g}_{1} \mathfrak{g}_{1} \supseteq \mathrm{g}_{2} \mathrm{~g}_{2} \supseteq \cdots \supseteq \mathrm{g}_{t} \mathrm{~g}_{\mathrm{t}} .
$$

These ideals are uniquely determined by \&, and we shall write $\mathfrak{R}=\left(\mathfrak{g}_{1}, \cdots, \mathfrak{g}_{t}\right)$. For any canonical splitting (1), we also have

$$
\mathfrak{R}=\left(\mathfrak{g}_{1} L_{1}\right) \perp \cdots \perp\left(\mathfrak{g}_{t} L_{t}\right) .
$$

Now if $\boldsymbol{X}$ is any subset of $L$, we define the invariant closure $\mathfrak{I}(X)$ to be the smallest invariant lattice containing $X$. It can be shown that

$$
\mathfrak{I}(X)=\left(\mathfrak{g}_{1}, \cdots, \mathfrak{g}_{t}\right) \text {, where } \mathfrak{g}_{i}=\varepsilon_{i}^{-1}\left(X \cdot L^{s_{i}}\right) \text { for } i=1, \cdots, t \text {. }
$$

3. Congruence subgroups. The mappings $X \rightarrow X$ and $X \rightarrow-X$ are both in $\mathrm{Sp}(L)$ and we denote them, when convenient, by +1 and -1 , respectively. For $\delta= \pm 1$ and $\sigma \in \operatorname{Sp}(L)$, we define ideals $o_{\delta, j}^{t}(\sigma)$, for $i, j=1, \cdots, t$, by

$$
\mathfrak{I}\left((\sigma-\delta) L^{s_{i}}\right)=\left(o_{\delta, 1}^{i}(\sigma), \cdots, \delta_{\delta, t}^{\delta}(\sigma)\right),
$$

and we put

$$
o_{j}^{i}(\sigma)=o_{+1, j}^{i}(\sigma) \cap o_{-1, j}^{i}(\sigma), \quad i, j=1, \cdots, t .
$$

It is convenient to think of the $t^{2}$ ideals $\left\{o_{j}^{i}(\sigma)\right\}$ as an array or matrix with the superscript as column index and the subscript as row index, and we define this array to be the order $o(\sigma)$ of $\sigma$. If we put $g_{j}^{t}=o_{\delta, t}^{t}(\sigma)$ (respectively, $g_{j}^{i}=o_{j}^{i}(\sigma)$ ), for $i, j=1, \cdots, t$, it can be shown that

$$
\begin{aligned}
& \mathfrak{g}_{j}^{j} \subseteq \mathfrak{D} \cap \mathbb{g}_{i} \mathbb{g}_{j}^{-1}, \quad \mathbb{g}_{i} \mathfrak{g}_{i}^{j}=\xi_{j} \dot{g}_{j}^{j}, \\
& \mathfrak{g}_{j}^{1} \supseteq \mathfrak{g}_{j}^{2} \supseteq \cdots \supseteq \mathfrak{g}_{j}^{t}, \quad g_{1}^{-1} \mathfrak{g}_{j}^{1} \subseteq g_{2}^{-1} \mathfrak{g}_{j}^{2} \subseteq \cdots \subseteq g_{i}^{-1} \mathfrak{g}_{j}^{t}, \\
& \mathfrak{g}_{1}^{i} \subseteq \mathfrak{g}_{2}^{i} \subseteq \cdots \subseteq \mathfrak{g}_{t}^{i}, \quad \dot{B}_{1} \mathfrak{g}_{1}^{i} \supseteq \boldsymbol{g}_{2} \mathfrak{g}_{2}^{i} \supseteq \cdots \supseteq g_{t} \mathfrak{g}_{t}^{i},
\end{aligned}
$$

for $i, j=1, \cdots, t$. Any set $\left\{g_{j}^{i}\right\}$ of $t^{2}$ ideals which satisfies (3) is called a tableau.

We can now define two particular congruence subgroups. The special congruence subgroup $\mathrm{SSp}=\mathrm{SSp}\left(L ;\left\{g_{j}^{i}\right\}\right)$ corresponding to the tableau $\left\{g_{j}^{i}\right\}$ consists of all $\sigma \in \operatorname{Sp}(L)$ with $o_{1, j}^{i}(\sigma) \subseteq g_{j}^{i}$ for all $i$ and $j$, while the general congruence subgroup $\mathrm{GSp}=\mathrm{GSp}\left(L ;\left\{g_{j}^{i}\right\}\right)$ consists of those $\sigma$ with $o_{j}^{i}(\sigma) \subseteq g_{j}^{i}$ for all $i$ and $j$. We have SSp $\subseteq$ GSp and both are normal subgroups of $\mathrm{Sp}(L)$.

Now take a canonical splitting (1). Define $\eta(i) \in \operatorname{Sp}(L)$ for $i=1, \cdots, t$ as follows: $\eta(i)\left|L_{i}=-1, \eta(i)\right| L_{j}=1$ for $j \neq i$. Let $\mathrm{H}$ be 
the group generated by $\eta(1), \cdots, \eta(t)$. Clearly $\mathrm{H}$ is abelian of type $(2,2, \cdots, 2)(t$ times). Then we define a homomorphism

$$
\Delta: \mathrm{GSP} \rightarrow \mathrm{H}
$$

using the order functions $o_{\delta, j}^{i}(\sigma)$. The kernel of $\Delta$ is SSp. The image of $\Delta$ is a subgroup $\mathrm{E}$ of $\mathrm{H}$.

THEOREM 2. If GSp and SSp are the congruence subgroups corresponding to a tableau $\left\{g_{j}^{i}\right\}$, then GSp is the semidirect product of $\mathrm{SSp}$ by the group $\mathrm{E}$ defined above. Thus $\mathrm{GSp} / \mathrm{SSp} \cong \mathrm{E}$, an abelian group of type $(2, \cdots, 2)$ (s times, $s \leqq t$ ). If $G$ is a subgroup of GSp which contains $\mathrm{SSp}$, then $G$ is the semi-direct product of $\mathrm{SSp}$ by a subgroup of $\mathrm{E}$.

4. Generation of SSp. In the local ring (and field) theory, the classical symplectic transvections generate the "special" congruence subgroups. In the present situation this is no longer true (although they do generate the symplectic group itself). In analogy to the orthogonal mappings defined by Siegel on p. 237 of [7], and also used in [4], we introduce the double transvection, of which the transvection is a special case: let $A$ and $C$ be in $L, A \cdot C=0$, and let $\lambda$ be in the field of quotients of $\mathfrak{v}$; if

$$
X \rightarrow X+\lambda(A \cdot X) C+\lambda(C \cdot X) A
$$

carries $L$ into itself, it is easy to check that it is in $\mathrm{Sp}(L)$, and we denote it by $\mathrm{T}_{A, C, \lambda}$. It is said to be pure if the pair $\{A, C\}$ can be extended to a canonical basis of $L$, and if $A=C$ when $A \cdot L=C \cdot L$.

TheOREM 3. The group $\operatorname{SSp}\left(L ;\left\{\mathrm{g}_{j}^{i}\right\}\right)$ is generated by the pure double transvections in it.

5. Main result. For any nonempty subset $G$ of $\operatorname{Sp}(L)$, we define $o(G)$ to be the tableau $\left\{\mathfrak{g}_{j}^{i}\right\}$ given by

$$
\mathfrak{g}_{j}^{i}=\max \left\{o_{j}^{i}(\sigma): \sigma \in G\right\} \text {. }
$$

Using Theorem 3 , we then prove

THEOREM 4. If $G$ is a normal subgroup of $\operatorname{Sp}(L)$ with $o(G)=\left\{g_{j}^{s}\right\}$, then

$$
\operatorname{SSp}\left(L ;\left\{g_{j}^{i}\right\}\right) \subseteq G \subseteq \operatorname{GSp}\left(L ;\left\{g_{j}^{i}\right\}\right) .
$$

Conversely, if (4) holds, then $G$ is normal and $o(G)=\left\{g_{j}^{i}\right\}$.

6. Normal subgroups as congruence subgroups. We now wish to describe the normal subgroups of $\operatorname{Sp}(L)$ by means of congruences. Choose a canonical basis for $L$ and let (1) be the corresponding 
canonical splitting. If $\sigma \in \operatorname{Sp}(L)$, the matrix $\operatorname{mat}(\sigma)$ of $\sigma$ in the canonical basis breaks up in an obvious manner into $t^{2}$ blocks $B_{f}^{i}$ $(i, j=1, \cdots, t)$ according to (1). If $\operatorname{mat}(\sigma)=\left(s_{\mu \nu}\right)$ and $\operatorname{mat}(\rho)$ $=\left(r_{\mu \nu}\right)$, we say that

$$
\sigma \equiv \rho \bmod \left\{g_{j}^{i}\right\}
$$

if $s_{\mu \nu} \equiv r_{\mu \nu} \bmod g_{j}^{l}$ for all $\mu$ and $\nu$, where $s_{\mu \nu}$ belongs to the block $B_{j}^{t}$. Now let $G$ be a normal subgroup of $\operatorname{Sp}(L)$ with $o(G)=\left\{g_{j}^{i}\right\}$. Using Theorem 2 and Theorem 4, one can show that there is a subgroup $\Gamma$ of $\mathrm{E}$ such that ${ }^{2}$

$$
G=\left\{\sigma \in \operatorname{Sp}(L): \sigma \equiv \gamma \bmod \left\{\mathfrak{g}_{j}^{\prime}\right\} \text { for some } \gamma \in \Gamma\right\} .
$$

Conversely, given a tableau $\left\{g_{j}^{i}\right\}$ and a subgroup $\Gamma$ of the group $E$ corresponding to $\left\{g_{j}^{2}\right\}$, the group defined by (5) is normal and has order $\left\{g_{j}^{i}\right\}$. An interesting special case is $\Gamma=\{1\}$; then $G$ $=\operatorname{SSp}\left(L ;\left\{\mathfrak{g}_{j}^{i}\right\}\right.$.

\section{BIBLIOGRAPHY}

1. E. Artin, Geometric algebra, Interscience, New York, 1957.

2. H. Bass, M. Lazard et J.-P. Serre, Sous-groupes d'indice fini dans $S L(n, Z)$, Bull. Amer. Math. Soc. 70 (1964), 385-392.

3. N. Bourbaki, Algèbre, Ch. 9, Hermann, Paris, 1959.

4. M. Eichler, Quadratische Formen und Orthogonale Gruppen, Springer-Verlag, Berlin, 1952.

5. W. Jehne, Die Struktur der symplektischen Gruppe über lokalen und dedekindschen Ringen, S. -B. Heidelberger Akad. Wiss., Springer-Verlag, Berlin, 1962/64.

6. W. Klingenberg, Symplectic groups over local rings, Amer. J. Math. 85 (1963), 232-240.

7. C. L. Siegel, Über die analytische Theorie der quadratischen Formen. II, Ann. of Math. (2) 37 (1936), 230-263.

\section{UNIVERSITY OF Notre DAME}

2 According to the definition of $\mathbf{E}$ given in $\$ 3$, the matrices of its elements in the canonical basis are diagonal matrices with 1's and -1 's on the diagonal; and the diagonal entries in any particular block $B_{i}^{i}$ are all 1 or all -1 . By way of comparison, in [6], the group $\mathbf{E}$ consists of \pm identity. 